\title{
EVALUATION OF DEBLOCKING METHODS FOR CHANG'E-4 DESCENT IMAGES
}

\author{
M. Peng, W. Wan*, Z. Liu, Y. Wang, K. Di \\ State Key Laboratory of Remote Sensing Science, Aerospace Information Research Institute, Chinese Academy of Sciences, Beijing, \\ China - (pengman, wanwh, liuzq, wangyx, dikc)@aircas.ac.cn
}

\author{
Commission III, ICWG III/II
}

KEY WORDS: Chang'e-4, descent camera image, image deblocking, fast adaptive bilateral

\begin{abstract}
:
Chang'e-4 lunar probe has successfully landed on the far side of the moon in Von Kármán crater inside the South Pole-Aitken (SPA) basin at 10:26 am on January 3, 2019. Due to the reduction of the coding rate, obvious block effects appear at the boundaries of descent images. Unblock, adaptive fast bilateral filtering, structure-texture enhancement and high-order Markov random field methods, are applied to remove the block effect of the descent images. Based on analysing the quality of descent images, quantitative comparison of four methods is performed using simulated compressed 1:64 descent images and real images. Comprehensive analysis was performed using typical measures such as PSNR, SSIM and NIQE. Experimental results show that adaptive fast bilateral filtering is better than other methods. The deblocked 1:64 image sequences have been used to assist localizing the landing point quickly during the mission.
\end{abstract}

\section{INTRODUCTION}

Chang'e-4 lunar probe has successfully landed on the far side of the moon in Von Kármán crater inside the South Pole-Aitken (SPA) basin at 10:26 am on January 3, 2019 (Di et al., 2019). As Chang'e-4 landed on the far side of the moon and was limited by the bandwidth of the "Queqiao" relay transmission link, the near-real-time, frame-framing, and compression method of the detector's power decline phase adopted a series of high-compression (1:64) JPEG compressed images. Due to the reduction of the coding rate, obvious block effects appear at the boundaries of image blocks. In order to perform subsequent lander localization and mapping, it is necessary to remove the block effect of descent camera images to improve the image quality.

The deblocking algorithms mainly include image enhancement and image restoration. The methods based on image enhancement smooth the block effect by filtering the image (Foi et al., 2007; Yoo et al., 2014; Li et al., 2014; Gavaskar et Chaudhury, 2018). Costella (2006) proposed the UnBlock algorithm without requiring the encoded JPEG or MPEG source file and any tunable parameters. A parabolic model is used to extrapolate blocking discontinuity artifacts in the gradient of the intensity. Based on the shape-adaptive discrete cosine transform (SA-DCT), Foi (2007) used the SA-DCT in conjunction with the anisotropic local polynomial approximation for denoising and deblocking. Yoo et al. (2014) presented a post processing framework for blocking artifact reduction, in which the interblock DCT coefficients for the three lowest frequency are smoothed to reduce blocking artifacts in the flat region, and 3-D filtering is applied to the edge region to reduce the remaining artifacts. Li et al. (2014) proposed a structure-texture enhancing method, which decomposes the input image into a structure layer and texture (high-frequency) layer, and contrast enhancement is then used for structural layer data. Gavaskar and Chaudhury (2018) proposed an efficient algorithm for adaptive bilateral filtering based on the observation that the concerned filtering can be performed purely in range space using an appropriately defined local histogram. In general, image enhancement approach aims at smoothing visible artifacts instead of restoring original pixel values, and it is somewhat heuristic in the sense that no objective criterion is optimized. The main advantage of this kind of approach is its relatively low computational complexity.

The image restoration-based algorithm treats the removal of block effects as the reconstruction of the image, and combines the prior information in the image to restore the image under the constraint of the maximum posterior criterion (Yang et al., 1995). Classical representative methods are iterative methods based on maximum posterior probability and algorithms based on convex set projection (Yang et al., 1997). Recently, learningbased sparse representation has been used for image deblocking. Jung et al. (2012) obtained an over-completed dictionary using the K-Singular Value Decomposition (K-SVD) algorithm, and proposed a new method to automatically estimate the residual threshold for Orthogonal Matching Pursuit (OMP). The enhanced images are obtained after solving objective function. Instead of processing each image patch individually, Zhang et al. (2015) proposed Group-based Sparse Representation (GSR) and a novel image deblocking method that was based on GSR and Quantization Constraint (QC) prior. Similarly, Zhao et al. (2017) proposed Structural Sparse Representation (SSR) and a novel algorithm for image deblocking was introduced by combining SSR with QC prior. All of them demonstrated that sparse representation could effectively remove image artifacts and ensure the image visual quality. Methods of sparse representation are based on compressed images of standard image libraries. The computational complexity of these methods is high because of their iterative processes.

Due to the lack of texture in lunar surface images, especially the Chang'e-4 descent images captured at high solar elevation angle, the performance of the deblocking method should be particularly considered. Therefore, in this paper, unblock

\footnotetext{
* Corresponding author.
} 
(Costella, 2006), adaptive fast bilateral filtering (Gavaskar and Chaudhury, 2018), structure-texture enhancement (Li et al., 2014), and high-order Markov random field (Sun et al., 2007) are chosen to remove the block effects in the images, and the performances of these methods are compared.

The rest of this paper is structured as follows: Section 2 analyses the characteristics of the compressed descent images The chosen four methods are presented in Section 3. Section 4 presents the experimental results. Finally, conclusions and suggestions for future work are given in Section 5.

\section{DATA}

A downward-looking descent camera identical to that on Chang'e- 3 is mounted on the bottom of Chang'e- 4 lander. The field of view (FOV) is $45.4^{\circ}$ with the image size of $1024 \times 1024$ pixels (Liu et al., 2015). The camera took a series of images during the descending process. However, because of the limitation of the communication bandwidth, the strategies of selective sampling and image compression with a ratio of 1:64 were used when transferring the descent images to the ground teleopration center for a quick view. During the power down phase, 59 images with 1:64 compression ratio are downlinked. After the separation of the lander and rover successfully completed, the probe sent back about 5441 original images of the landing, including about 5,300 1:8 low compression ratio images. These data are used for accurate positioning of the landing point and high-resolution mapping of the landing site. Figure 1(a) show the first descent image of 3000 meters above the lunar surface, Figure 1(b) show the first landing image with a ratio of $1: 8$

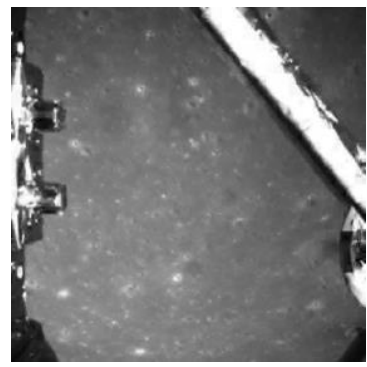

(a) The first descent image (2019-01-03T10:23:03, Beijing time)

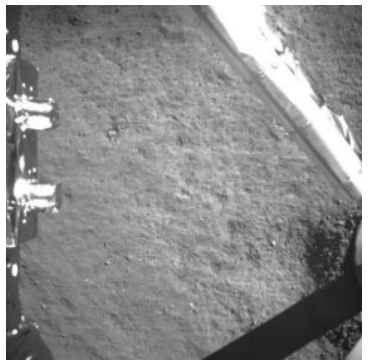

(b) The first landing image (2019-01-03T11:44:33, Beijing time)
Figure 1. The first descent image and landing image received by the ground teleoperation center

In order to enhance the 1:64 compressed image effectively, four measurements including the average gray value, standard deviation (std) of image, entropy and anisotropy are calculated to analyse the quality of 87 descent images within 100 meters above the lunar surface. The entropy and anisotropy are defined as

$$
\begin{gathered}
\text { entropy }=-\sum_{0}^{255} \operatorname{rel}[i] * \log _{2}(\operatorname{rel}[i]) \\
\text { Anisotropy }=\frac{\sum_{0}^{k} r e l[i] * \log _{2}(\operatorname{rel}[i])}{\text { Entropy }}
\end{gathered}
$$

where $\operatorname{rel}[\mathrm{i}]$ represents the histogram, i represents the gray value in $[0,255], \mathrm{k}$ is the smallest possible gray value with $\operatorname{sum}(\operatorname{rel}[\mathrm{i}])>=0.5$. In general, higher entropy value represents higher image quality. The anisotropy describes the characteristics of gray value changing with different directions. The smaller of the anisotropy value, the poorer the texture is.

The statistical results show that the minimum value of mean gray value is 100.9 , the maximum value is 137.1 , which show that the range of gray value is small. This is reflected in Figure 1 that the descent images are of low texture. The entropy value reflects the image information, as the images are all near the landing site so that the entropy of descending images changes little. For the same reason, the difference between the minimum value and maximum value of anisotropy is also very low.

\begin{tabular}{|c|c|c|c|c|}
\hline & $\begin{array}{c}\text { Mean } \\
\text { gray }\end{array}$ & std & entropy & $\begin{array}{c}\text { anisotr } \\
\text { opy }\end{array}$ \\
\hline maximum & 137.1 & 47.6 & 7.2 & -0.489 \\
\hline minimum & 100.9 & 43.6 & 6.7 & -0.529 \\
\hline median & 103.8 & 45.4 & 7.1 & -0.515 \\
\hline
\end{tabular}

Table 1. Statistical results of descent images

\section{METHODS}

Four methods including unblock, fast adaptive bilateral filtering, structure-texture enhancing, and high-order Markov random field (MRF) and applied to compare the performance of deblocking. The first three methods are image enhancing algorithms, while the last one is an image restoration method.

\subsection{Unblock algorithm}

Being different of most deblocking methods which are mathematically complicated, unblock algorithm is a postprocessimg method without requiring the encoded JPEG or MPEG source file (Costella, 2006). It measures the discontinuity in each component's value across the boundary, and the discontinuity is smoothed out based on some criteria. Firstly, a linear variation of intensity on each side of a boundary is assumed, next the amount of compression is adapted to determine the distribution of the magnitudes of the block boundary discontinuity. By computing the distribution of boundary discrepancies across the entire image, and comparing it to that of the interior of the blocks, the amount to which the compression has introduced block artifact can be quantified statistically. Finally, the intensity values of the pixels near the adjacent boundaries will be changed, and the boundary discrepancies are adjusted.

In order to measure the discrepancies at the central boundary, a parabolic model is used in each $8 \times 8$ pixels region to measure discrepancies. The intensity discrepancy $u$ is defined as

$$
u=\frac{15\left(i_{9}-i_{8}\right)-10\left(i_{10}-i_{7}\right)+3\left(i_{11}-i_{6}\right)}{8}
$$

Moreover, the derivative discrepancy $v$ is

$$
v=-2\left(i_{9}+i_{8}\right)+3\left(i_{10}+i_{7}\right)-\left(i_{11}+i_{6}\right)
$$

By symmetrically dealing with the boundary pixels, the correction values of the sixteen pixel intensity are obtained.

$$
\begin{array}{llll}
\Delta i_{1}^{v}=-\frac{v}{256} & \Delta i_{2}^{v}=-\frac{11 v}{256} & \Delta i_{3}^{v}=-\frac{31 v}{256} & \Delta i_{4}^{v}=-\frac{58 v}{256} \\
\Delta i_{5}^{v}=-\frac{57 v}{256} & \Delta i_{6}^{v}=-\frac{22 v}{256} & \Delta i_{7}^{v}=+\frac{42 v}{256} & \Delta i_{8}^{v}=+\frac{138 v}{256} \\
\Delta i_{9}^{v}=+\frac{138 v}{256} & \Delta i_{10}^{v}=+\frac{42 v}{256} & \Delta i_{11}^{v}=-\frac{22 v}{256} & \Delta i_{12}^{v}=-\frac{57 v}{256} \\
\Delta i_{13}^{v}=-\frac{58 v}{256} & \Delta i_{14}^{v}=-\frac{31 v}{256} & \Delta i_{15}^{v}=-\frac{11 v}{256} & \Delta i_{16}^{v}=-\frac{v}{256}
\end{array}
$$




\subsection{Fast adaptive filter method}

Fast adaptive bilateral filtering (FABF) (Gavaskar and Chaudhury, 2018) is a fast algorithm for adaptive bilateral filtering, whose complexity does not scale with the spatial filter width. In addition, the concerned filtering can be performed purely in range space using an appropriately defined local histogram. The adaptive bilateral filter is

$$
g(i)=\eta(i)^{-1} \sum_{j \in \Omega} w(j) \phi_{i}(f(i-j)-\theta(i)) f(i-j)
$$

where

$$
\eta(i)=\sum_{j \in \Omega} w(j) \phi_{i}(f(i-j)-\theta(i))
$$

$\Omega$ is a window centered at the origin, $\phi_{i}$ is the local Gaussian range kernel.

$$
\phi_{i}(t)=\exp \left(-\frac{t^{2}}{2 \sigma(i)^{2}}\right)
$$

The spatial kernel $\omega$ is Gaussian function:

$$
\omega(j)=\exp \left(-\frac{\|j\|^{2}}{2 \rho^{2}}\right)
$$

and the center $\theta(i)$ and width $\sigma(i)$ are spatially varying functions.

\subsection{Structure-texture enhancing method}

Structure-texture enhancing method firstly decomposes the input image into a structure layer and texture (high-frequency) layer, contrast enhancement is then used for structural layer data (Li et al., 2014). The input image can be considered as the superimposition of the two layers:

$$
I_{i}=I_{s_{i}}+I_{T_{i}}
$$

where $I_{\mathrm{S}}$ represents main large objects in the image, and $I_{\mathrm{T}}$ represents fine details.

Based on the total-variation (TV) regularization, the structure layer $I_{\mathrm{S}}$ can be obtained by minimizing the following function

$$
\min _{I_{S}} \sum_{i}\left(I_{S_{i}}-I_{i}\right)^{2}+\lambda\left|\nabla I_{S_{i}}\right|
$$

where $i$ is the pixel index, $\lambda$ is the regularization parameter and $\nabla$ is the gradient operator.

Because texture layer data includes image details and block effects, block effects are removed from texture layer data. The objective function is defined as follows:

$$
\min _{I_{T_{i}}^{d}} \sum_{i}\left(I_{T_{i}}^{d}-I_{T_{i}}\right)^{2}+\beta \sum_{i \in \eta}\left(\nabla I_{T_{i}}^{d}\right)^{2}
$$

where $\eta$ are locations of the $8 \times 8$ pixels block borders. Since the block artifacts mostly appear on the edges, the function is used to smooth the edges at the borders.

The texture layer is enhanced by multiplication scale factor $K$, which is

$$
I_{T}^{e}=K \circ I_{T}^{d}
$$

where $I_{T}^{e}$ is the enhanced texture layer.

After proper scaling is completed, the processed texture layer data is added to the structural layer to generate the final enhanced image.

\subsection{High order Markov random field}

Sun et al. (2007) proposed a postprocessing method according to maximum a posteriori criterion. The image distortion is modeled as additive and spatially correlated Gaussian noise, and the original image is seen as high order Markov random field. After minimizing the energy function, the enhanced image can be obtained. The energy function is

$$
\begin{gathered}
E(I)=E_{i}(I)+\lambda E_{n}(I) \\
=-\sum_{k \in S} \sum_{i=1}^{N} \log \phi\left(J_{i}^{T} I_{c_{k}} ; \alpha_{i}\right)+\lambda \sum_{m} \frac{1}{2} n_{q}^{t}(m) \sum_{q}^{-1} n_{q}(m) \\
\phi\left(J_{i}^{T} I_{c_{k}} ; \alpha_{i}\right)=\left[1+\frac{1}{2}\left(J_{i}^{T} I_{c_{k}}\right)^{2}\right]^{-\alpha_{i}} \\
n_{q}(m)=I_{q}(m)-I(m)
\end{gathered}
$$

where $J_{\mathrm{i}}$ is a filter of size $n \times n, C_{k}$ includes $n \times n$ pixels with $k$ as center, $J_{i}^{T} I_{c_{k}}$ is the inner product between the filter and the local image patch, $S^{\prime}$ contains all the center pixels of the $n_{1} \times n_{1}$ cliques, $\alpha_{i}$ is a parameter related to $J_{\mathrm{i}}, N$ is the number of used filter. $\lambda \geq 0$ represents regularization parameter, $m$ is a block index, $n_{\mathrm{q}}(m), I_{\mathrm{q}}(m), I(m)$ represent quantization noise, the compression image and the original image respectively, where

$$
n_{q}(m) \sim \mathrm{N}\left(0, \Sigma_{q}\right)
$$

$\Sigma_{q}$ is a $64 \times 64$ invertible matrix, which can be determined from the DCT domain.

\section{EXPERIMENT}

To compare the performances of the deblocking methods, the experimental section consists of two parts: simulated images and real images. The simulated images are obtained by compressing the 1:8 descent images. In the quality assessment of the deblocking results, the original 1:8 image is used as reference image. The real images are chosen from 1:64 descent images, the corresponding experimental results are evaluated with several quantitative measures.

\subsection{Quantitative evaluation factors}

In order to evaluate the quality of the reconstruction image, we use the following five quantitative evaluation factors in the experiments: PSNR (peak signal-to-noise ratio), SSIM (structural similarity index), image contrast, image clarity and NIQE (Naturalness Image Quality Evaluator).

PSNR is very commonly used in the quantitative evaluation of image deblocking results and is based on the mean square error between the enhanced image and the reference image, with relation to the logarithmic of $\left(2^{\mathrm{t}}-1\right)^{2}$ (the maximum square of the signal), where $t$ is the number of bits for each pixel value. We generally use eight bits to represent each pixel, so the formula is

$$
P S N R=10 \times \log _{10} \frac{255^{2} \times m n}{\sum_{i=1}^{m n}\left(\hat{x_{i}}-x_{i}\right)^{2}}
$$

A higher PNSR value indicates a better deblocking performance.

SSIM is similar to the evaluation of the visual interpretation and is defined as 


$$
\operatorname{SSIM}=\frac{\left(2 u_{x} u_{\hat{x}}+C_{1}\right)\left(2 \sigma_{x \hat{x}}+C_{2}\right)}{\left(u_{x}^{2}+u_{\hat{x}}^{2}+C_{1}\right)\left(\sigma_{x}^{2}+\sigma_{\hat{x}}^{2}+C_{2}\right)}
$$

where $u_{x}$ and $u_{\hat{x}}$ are the mean values of the original image and the deblocked image, respectively. $\sigma_{x}$ and $\sigma_{\hat{x}}$ represent the variance of the original image and the deblocked results, respectively. $\sigma_{x \hat{x}}$ is the covariance between the original image and the deblocked image. $\mathrm{C}_{1}$ and $\mathrm{C}_{2}$ are constants to prevent the equation from being meaningless (numerator and denominator not equal to zero). SSIM is an evaluation factor used to characterize the contrast, brightness, and structural similarity of an image. It ranges from $0-1$, and the closer to 1 , the better the image quality is.

For images without reference images, image contrast can be computed from the derivation between the value of each pixel and the mean gray value. The image clarity is defined as the gray value difference of neighbour pixels, which is

$$
\text { clarity }=\sum_{x} \sum_{y}[f(x, y)-f(x+1, y)] *[f(x, y)-f(x+1, y)]
$$

The higher of the value, the clearer of the deblocked image is.

Furthermore, NIQE is also calculated to evaluate the nonreference image quality. It is based on the construction of a 'quality aware' collection of statistical features, which can be obtained from a simple and successful space domain natural scene statistic (NSS) model (Mittal et al., 2013). A smaller score indicates better perceptual quality. The score is expressed as the distance between the quality aware NSS (natural scene statistic) feature model and the MVG (multivariate Gaussian) model fit to the features extracted from the deblocked images,

$$
N I Q E=\sqrt{\left(\left(v_{1}-v_{2}\right)^{T}\left(\frac{\sum_{1}+\sum_{2}}{2}\right)^{-1}\left(v_{1}-v_{2}\right)\right)}
$$

where $v 1, v 2$ and $\Sigma_{1}, \Sigma_{2}$ are the mean vectors and covariance matrices of the natural MVG model and deblocked image's MVG model.

\subsection{Experiments of simulatied images}

Simulated 1:64 compressed images are generated from 1:8 descent images. Experiments were conducted using 50 simulated 1:64 compressed images. Figure 2 shows an example of simulated image using four deblocking methods. The enlarged view of Figure 2(b) show distinct block effect, especially the edges between adjacent blocks in craters affect the image quality. Figure 2(c) and (d) show enhanced image while removing the edges. It is noted that details in Figure 2(e) are blurred compared with original image. The edges in Figure 2 (f) are still distinct.

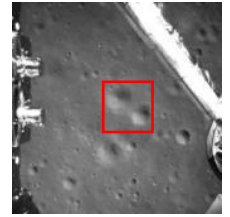

(a) simulated 1:64 compressed image

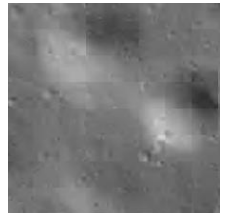

(b) enlarged view of the area outlined by a rectangle in (a)

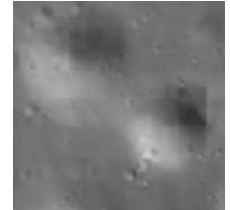

(c)enhanced results using unblock method

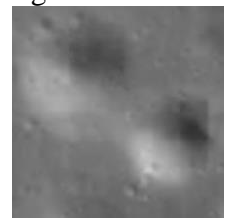

(e) enhanced results using structure-texture method

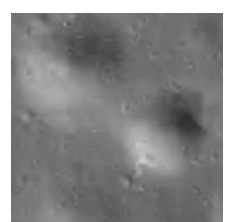

(d) enhanced results using FABF filtering method

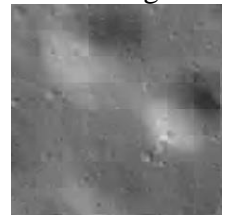

(f) enhanced results using high-order MRF method

Figure 2. Visual quality comparison of simulated 1:64 compressed image

\begin{tabular}{|c|c|c|c|c|}
\hline Method & contrast & clarity & PSNR & SSIM \\
\hline unblock & 45.550 & 3.291 & 27.935 & 0.839 \\
\hline FABF & 45.886 & 3.373 & 28.026 & 0.843 \\
\hline $\begin{array}{c}\text { structure } \\
\text {-texture }\end{array}$ & 44.682 & 3.182 & 27.764 & 0.837 \\
\hline MRF & 45.468 & 2.340 & 27.340 & 0.830 \\
\hline
\end{tabular}

Table 2. Quality metrics of deblocked simulated images

Table 2 lists the mean quality metrics of deblocked simulated images. The contrast of four kinds of results are close to each other, with the MRF deblocking method getting the lowest clarity. PSNR and SSIM are calculated based on the original 1:8 compressed images, FABF shows the highest value. Overall, FABF method gets the best performance among the four methods.

\subsection{Experiments of real images}

Real data experiments were conducted using 87 Chang'e-4 compressed 1:64 images. Figure 3 shows an example of descent images using four deblocking methods. Figure 3(b) shows an enlarged view of the area outlined by a red rectangle in (a). The artifacts located at the rim of crater are obvious. After applying the four methods, the image has been enhanced. However, there are still some edges in Figure 3(f). Figure 3 (c) and (d) show enhanced results, while Figure 3 (e) enhanced the image at the expense of smoothing the texture.

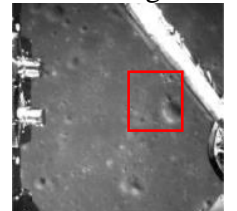

(a) 1:64 descending image

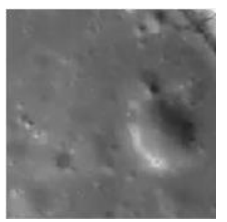

(c) enhanced results using unblock method

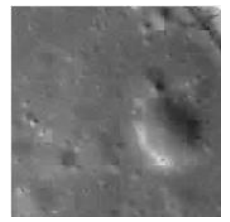

(b) enlarged view of the area outlined by a rectangle in (a)

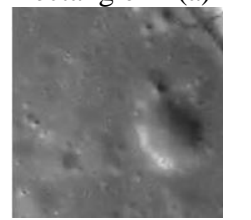

(d) enhanced results using FABF method 


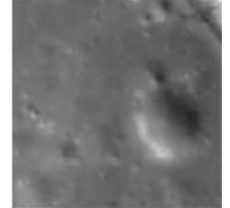

(e) enhanced results using structure-texture method

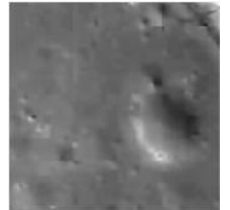

(f) enhanced results using high-order MRF method
Figure 3. Visual quality comparison of 1:64 compressed image

\begin{tabular}{|c|c|c|c|}
\hline Method & contrast & clarity & NIQE \\
\hline unblock & 45.259 & 2.477 & 5.379 \\
\hline FABF & 45.318 & 2.667 & 5.099 \\
\hline $\begin{array}{c}\text { structure- } \\
\text { texture }\end{array}$ & 44.464 & 2.050 & 5.030 \\
\hline MRF & 44.312 & 2.023 & 5.521 \\
\hline $\begin{array}{c}\text { Original 1:64 } \\
\text { descending } \\
\text { image }\end{array}$ & 42.206 & 1.958 & 5.637 \\
\hline
\end{tabular}

Table 3. Quality metrics of deblocked descent images

Table 3 lists the mean quality metrics of deblocked descent images. As there is no reference image, NIQE is adopted instead of PSNR and SSIM. FABF gets the lowest value which represents best performance. Being similar with the results of simulated images, fast adaptive bilateral method gets the best performance among the four methods. The results of real data experiments show good agreement with that of simulated data.

The deblocked 1:64 image sequences have been used to localize the landing point quickly during the mission (Wang et al., 2020), and the localization results are used as an initial value to get the final results based on playback 1:8 compressed images.

\section{CONCLUSION}

In this paper, an evaluation and comparison of deblocking methods for Chang'e- 4 descent images have been presented. We focused on verifying the deblocking ability of different algorithms that could be used in enhancement of lunar images. Based on analysing the quality of descent images, quantitative analysis of four methods is performed using simulated compressed 1:64 descent images and real images. Comprehensive analysis was performed using typical measures such as PSNR, SSIM and NIQE. In experiments of simulated images, the results of PSNR and SSIM show that FABF is better than the other methods. Further experiments of real descent images verified the former conclusion. The results can be used as guidelines in the design of deblocking method for planetary images.

\section{ACKNOWLEDGEMENTS}

This study was supported by National Natural Science Foundation of China under Grants 41771488 and 41701489.
Di, K.C., Liu, Z.Q., Liu, B., Wan, W.H., Peng, M., Wang, Y.X., Gou, S., Yue, Z.Y., Xin, X., Jia, M.N., Niu, S.L., 2019. Chang'e-4 lander localization based on multi-source data. Journal of Remote Sensing, 23(1): 177-184.

Foi, A., Katkovnik, V., Egiazarian, K., 2007. Pointwise shapeadaptive DCT for high-quality denoising and deblocking of grayscale and color images. IEEE Transactions on Image Processing, 16(5): 1395-1411.

Gavaskar, R.G., Chaudhury, K.N., 2018. Fast adaptive bilateral filtering. IEEE Transactions on Image Processing, 28(2), 779790.

Li, Y., Guo, F., Tan, R.T., Brown, M.S., 2014. A contrast enhancement framework with JPEG artifacts suppression. European Conference on Computer Vision, 174-188.

Liu, Z.Q, Di, K.C., Peng, M., Wan, W.H., Liu, B., Li, L., Yu, T., Wang, B.F., Zhou, J.L., Chen, H., 2015. High precision landing site mapping and rover localization for Chang'e-3 mission. Science China-Physics Mechanics \& Astronomy, 58, 1-11.

Mittal, A., Soundararajan, R, Bovik, A.C., 2013. Making a Completely Blind Image Quality Analyzer. IEEE Signal Processing Letters, 20(3): 209-212.

Sun, D., Cham, W., 2007. Postprocessing of Low Bit Rate Block DCT Coded Images based on a Fields of Experts Prior. IEEE Transactions on Image Processing, 16(11), 2743-2751.

Wang, J., Wu, W.R., Li, J., Di, K.C., Wan, W.H., Xie, J.F., Peng, M., Wang, B.F., Liu, B., Jia, M.N., Xi, L.H., Zhao, R., 2020. Vision based Chang'E-4 landing point localization (in Chinese). SCIENTIA SINICA Technologica, 50: 41-53,

Yang, Y., Galatsanos, N.P., Katsaggelos, A.K., 1995. Projection-based spatially adaptive reconstruction of blocktransform compressed images. IEEE Trans. Image Process, 4(7): 896-908.

Yang, Y. Galatsanos, N.P., 1997. Removal of compression artifacts using projections onto convex sets and line process modelling. IEEE Trans. Image Process. 6(10): 1345-1357.

Yoo, S.B., Choi, K., Ra, J.B., 2014. Post-processing for blocking artifact reduction based on inter-block correlation. IEEE Transactions on Multimedia, 1536-1548.

Zhang, J., Ma, S., Zhang, Y., Gao, W., 2015. Image deblocking using group-based sparse representation and quantization constraint prior. International conference on Image processing, 306-310.

;

Zhao C, Zhang J, Ma S, Fan, X., Zhang, Y., Gao, W., 2017. Reducing Image Compression Artifacts by Structural Sparse Representation and Quantization Constraint Prior. IEEE Transactions on Circuits and Systems for Video Technology, 27(10): 2057-2071.

\section{REFERENCES}

Costella, J.P., 2006. The unblock algorithm. 